\title{
Proyecto integrado para enseñar ciencias en bachillerato: Organización y gestión de un centro hospitalario
}

\author{
María del Mar Estévez Ferrer, Alicia Fernández Oliveras y Francisco Javier Carrillo Rosúa. \\ Universidad de Granada \\ 口 \\ Recepción: 12 de abril de 2015 | Revisión: 30 de mayo de 2015 | Aceptación/Publicación: 24 de julio de 2015 \\ Correspondencia: fjcarril@ugr.es | alilia@ugr.es | http://hdl.handle.net/10481/37123
}

Resumen: Este artículo recoge una propuesta de enseñanza con un enfoque competencial y globalizador, basada en el aprendizaje activo y cooperativo: un proyecto integrado sobre la gestión de un centro hospitalario. La metodología de proyectos posibilita que el alumnado dirija la evolución de su aprendizaje, fomentando que los adolescentes se incorporen a la vida adulta en condiciones de aplicar sus conocimientos, argumentar sus opiniones e integrarse positivamente en la sociedad. Como ventaja adicional de esta metodología de enseñanza, podemos señalar el desarrollo de las capacidades emprendedoras que la actualidad está demandando y que creemos imprescindible fomentar desde el ámbito educativo. La implementación en $2^{\circ}$ curso de Bachillerato del proyecto integrado propuesto, que integra conocimientos científicos con conciencia social y ambiental, permitirá al alumnado poner en juego aprendizajes adquiridos a lo largo de todo su periodo educativo y favorecerá el desarrollo de futuros proyectos laborales o académicos donde se puedan retomar las competencias ya adquiridas. También podría abordarse un proyecto de este tipo como acción extracurricular con fines de alfabetización científica. Por ello, consideramos que esta propuesta puede resultar de interés para la formación de profesionales de la educación tanto formal como no formal.

Palabras clave: Didáctica de las Ciencias Experimentales | Proyecto Integrado | Acción Extracurricular | Enseñanza Globalizadora | Enfoque Competencial

INTEGRATED PROJECT FOR TEACHING SCIENCE IN HIGH SCHOOL: ORGANIZATION AND MANAGEMENT OF A HOSPITAL

Abstract: This article contains a teaching proposal using a competence-oriented globalizing approach, based on active and cooperative learning: an integrated project about the management of a hospital. The project's methodology enables students to steer their learning path, encouraging them to become part of adult life. Thus, adolescents may be able to apply their own knowledge, to back up their opinions with arguments and positively integrate into society. An additional advantage of this teaching methodology is the development of entrepreneurial skills that today's world is demanding and, in our view, essential to promote this in an educational environment. The implementation of the project proposed, which brings together scientific knowledge and social and environmental awareness, will allow students in their final year of secondary school to apply learning acquired throughout their studies. Moreover, it will encourage the development of future academic and professional projects where they can apply the skills already acquired. This kind of project may also be regarded as an extracurricular activity involving scientific literacy. Therefore, we believe that this proposal may be of interest for training professionals in formal and non-formal education.

Keywords: Science Education | Integrated Project | Extracurricular Activity | Globalizing Education | Competence Approach

\section{Introducción}

En el presente artículo se pretende realizar una propuesta de enseñanza con un enfoque competencial y globalizador, basada en el aprendizaje activo y cooperativo: un proyecto integrado (Hernández de La Torre, 2010; Lozano, 1998; Torres, 2000) dirigido al $2^{\circ}$ curso de Bachillerato.

La materia optativa Proyecto Integrado, que se incluía en Andalucía de acuerdo con la Orden de 5 agosto de 2008 (Consejería de Educación, 2008) con el objetivo de que el alumnado pusiera en juego los conocimientos adquiridos en las distintas materias cursadas previamente para desarrollar las competencias básicas, desaparece del currículo oficial con la entrada de la Ley Orgánica 8/2013 de 9 de diciembre, para la Mejora de la Calidad Educativa (Ministerio de Educación, Cultura y Deporte, 2013). Así, en el Real Decreto que lleva a cabo el desarrollo legislativo de esta ley, no existe una asignatura específica que siga una metodología de aprendizaje por proyectos (Ministerio de Educación, Cultura y Deporte, 2015a) y aun 
no se ha publicado el desarrollo legislativo en Andalucía, que pudiera darle cabida.

En cualquier caso, sí habría posibilidad de utilizar esta metodología en cualquiera de las asignaturas ofertadas, ya que en el Artículo 29, referido a procesos de aprendizaje, podemos encontrar lo siguiente (Ministerio de Educación, Cultura y Deporte, 2015a, p. 24): "Las actividades educativas en el Bachillerato favorecerán la capacidad del alumnado para aprender por sí mismo, para trabajar en equipo y para aplicar los métodos de investigación apropiados", lo que podemos considerar como base del aprendizaje por proyectos. Y la Ley Orgánica 8/2013, en su artículo 6bis (Ministerio de Educación, Cultura y Deporte, 2013) establece la existencia de asignaturas de libre configuración autonómica que podrían tener una metodología equiparable a la de Proyecto Integrado, siendo esto competencia de las Comunidades Autónomas y de los Centros Educativos que tienen la potestad de diseñar e implantar métodos pedagógicos y didácticos propios. También podría abordarse un proyecto de este tipo como parte de una acción extracurricular con fines de alfabetización científica, bien durante el curso o bien en periodos vacacionales, por ejemplo, en el marco de iniciativas semejantes a los clubes de ciencias o a los campamentos científicos. Por ello, consideramos que nuestra propuesta puede resultar de interés para la formación de profesionales de la educación tanto formal como no formal.

Como argumento adicional a favor de la utilización de esta metodología de enseñanza, podemos señalar el desarrollo en las capacidades emprendedoras. Debido a la actual situación económica y laboral, y ante la falta de oportunidades para miles de jóvenes en el mercado laboral, es importante fomentar la cultura emprendedora entre los estudiantes. Es lo que la sociedad actual está demandando, la realización de proyectos y actividades que contribuyan a la generación de empleo e inversión, sobre todo en pequeñas y medianas empresas (PYMES). Y es que las PYMES son parte fundamental en el crecimiento económico de un país. El desarrollo del emprendimiento debe de ser una parte importante en la educación y la formación, ya que se considera uno de los principales factores para fomentar el espíritu empresarial. Siendo el conocimiento una herramienta para desarrollar iniciativas empresariales con más posibilidades de sobrevivir y crecer, es imprescindible que en el ámbito educativo español se fomente una cultura más emprendedora, que apoye el descubrimiento y desarrollo de oportunidades por los propios estudiantes. En este sentido, la implementación de un proyecto integrado dentro de las asignaturas de Bachillerato se presta a desarrollar tales objetivos.

En nuestra propuesta, el proyecto integrado será presentado como la gestión de un centro hospitalario en el que el alumnado participante se encarga de todas las áreas y ha de desarrollar procedimientos y proponer soluciones ante distintos problemas abiertos. Para ello, deberá poner en juego las competencias básicas y conocimientos adquiridos en las diferentes materias a fin de trabajar globalmente.

La elección de la temática del proyecto viene marcada porque en ella se integran muchos contenidos trabajados en las diferentes materias impartidas a lo largo de la Educación Secundaria, además del interés de que los estudiantes tomen conciencia de la realidad que les rodea y de los problemas existentes, en este caso, profundizando en el territorio de la sanidad y de todo lo que ésta conlleva, para poder ofrecer un buen servicio al ciudadano. Aunque se toquen todas las ramas de la Educación Secundaria, este proyecto está más encaminado a las materias de ciencias y sus aplicaciones. Se propondrá al alumnado la elaboración de actividades enmarcadas en el tema del proyecto, pero que sirvan para profundizar en las competencias clave desarrolladas durante la Educación Secundaria Obligatoria. 


\section{Marco teórico}

La implementación de un proyecto integrado promueve la imaginación, la innovación y la colaboración entre los estudiantes. Además de facilitar una integración más natural de los medios digitales y sociales, el alumnado trabaja directamente con la documentación aprendiendo a aprender y a trabajar autónomamente. Si se contextualiza dentro de un problema cuya resolución se implique el ámbito científico, aunque no tiene por qué ser estrictamente necesario, se puede conseguir el objetivo de hacer cercana y cotidiana la ciencia. Con esto se pretende ayudar al alumnado a que sea consciente de los conocimientos que tiene y como utilizarlos cuando la situación lo requiera, esto es, a desarrollar las competencias clave (Ministerio de Educación, Cultura y Deporte, 2015b).

Al decir "conocimientos" en general, estamos incluyendo tanto los conocimientos declarativos como los procedimentales y actitudinales. Se consideran conocimientos declarativos ("saber qué") aquellos relacionados con datos, hechos, conceptos y principios. Pozo (1992) los divide en conocimientos factuales y conceptuales. El conocimiento factual es el referido a datos y hechos que suministran información y que se pueden aprender literalmente, ya que no requiere de la comprensión. En cambio, el aprendizaje conceptual necesita apoyarse en conocimientos previos, para que se instaure el proceso de asimilación y comprensión. Los conocimientos procedimentales ("saber hacer") implican la ejecución de procedimientos, estrategias, técnicas, habilidades, destrezas y métodos. Están basados en la realización de acciones, por lo que remiten a conocimientos prácticos. El aprendizaje es una progresión gradual, desde una ejecución insegura, hasta llegar a una ejecución sin falla, consciente y casi automática. Los conocimientos actitudinales involucran juicios evaluativos. Reflejo de los valores que posee una persona, las actitudes se ponen de manifiesto y se aprenden en el contexto social (Díaz y Hernández, 1999).

La ventaja del aprendizaje basado en proyectos consistente en que la resolución de los problemas "reales" aportada puede ser válida y a la vez no tiene por qué ser necesariamente "la correcta" (no habrá, ni se ofrecerá una única solución posible); es un punto fuerte a la hora de motivar al alumnado, pues al existir varias posibilidades para las respuestas correctas, se reduce el miedo a la equivocación y al fracaso y se potencia la creatividad. Además, los proyectos provocan que los estudiantes se interroguen sobre las cosas y no se conformen con la primera respuesta, problematizando la realidad y abriendo así sus intereses. También permiten al alumnado diseñar procesos de trabajo activo y relacionarse de modo más independiente con el mundo natural, cultural y sociotecnológico que habita, ampliando su capacidad de participar conscientemente en la conducción del propio aprendizaje (La Cueva, 2006).

Por otro lado, hay que tener en cuenta el gran esfuerzo e implicación que conlleva la organización y puesta en marcha de un proyecto. El profesorado debe documentarse, relacionar conocimientos previos de diferentes áreas, diseñar actividades adecuadas y motivar al alumnado con lo planteado. Por ello, el éxito del proyecto depende, en gran medida, de la buena preparación y la actualización del profesorado que lo lleve a cabo (Porlán y Martín, 2008).

\section{Metodología}

El proyecto integrado se plantea como una serie de problemas abiertos que se van a proponer al alumnado a lo largo del curso. Se dará una definición de cada problema y se preverán los medios que permitan alcanzar una de las múltiples soluciones 
posibles. Para ello, se constituirán 6 grupos de trabajo diferentes en la temática que abordarán (sanitaria, ambiental, económica, infraestructuras, documentación medica y nutrición), pero homogéneos en tamaño. Conforme se vayan superando las actividades, los participantes rotarán por todos los grupos para trabajar en los distintos roles propuestos. Con dichos grupos se intenta que quede representada la totalidad de las funciones que se encuentran en un hospital de manera genérica. Los grupos deberán estar en constante comunicación pues todos están ligados y necesitan colaborar para la consecución de los objetivos.

Las actividades serán mensuales y, al principio de cada una de ellas, el profesorado debe exponer las incidencias particulares que afectan a los diferentes grupos de trabajo. Entonces, los integrantes de los grupos deben analizar los datos y llegar a conclusiones, proponer soluciones e indicar los materiales que se deben usar y el personal que interviene. Las sesiones se plantean como foros de discusión en los que los participantes tendrán la posibilidad de plantear al resto de los compañeros cualquier duda u opinión que deseen, lo cual facilita la creación de estrategias de aprendizaje en común.

La función de cada grupo de trabajo será la siguiente:

1. Grupo sanitario y de salud. El alumnado se enfrentará a problemas de carácter sanitario y de salud en casos reales o ficticios. Problemas tales como identificación de enfermedades según síntomas y signos en pacientes, interpretación de datos, búsqueda de orígenes o causas y propuestas de tratamientos.

2. Grupo ambiental. Los integrantes de este grupo abordarán la búsqueda de alternativas sostenibles a las instalaciones dadas por el profesorado y a la gestión de los residuos generados con cada caso propuesto, también deberán hacer frente a las incidencias que se puedan presentar en cada caso y donde la decisión del tipo de actuación que se tome estará a cargo del estudiante.

3. Grupo de gestión económica. Control de datos, argumentación y negociación con el resto de los grupos y toma de decisiones serán los retos que afrontará el alumnado al formar parte de este grupo. Es el grupo al que más deben tener en cuenta el resto para poder llevar a cabo las acciones y planes que deseen poner en marcha, pues sin el consentimiento de este grupo será imposible realizarlas, ya que se deben ajustar a un presupuesto cerrado en cada caso.

4. Grupo de infraestructuras y mantenimiento. En este grupo, el alumnado abordará temáticas relacionadas con las instalaciones, los equipos electrónicos que se emplean en las pruebas médicas, la seguridad y los accesos del hospital, también deben tomar decisiones a la hora de mejora, arreglo o cambio de la maquinaria que se esté empleando en cada actividad, se ocuparán de buscar alternativas sostenibles en cada caso.

5. Grupo de documentación médica. Los miembros de este grupo se ocuparán de la informatización de las historias médicas, los resultados de las pruebas y los datos del personal del hospital.

6. Grupo de nutrición. La función principal del alumnado será dirigir la elaboración de los menús ofertados por la cafetería-restaurante donde tanto los familiares de internos como el personal del hospital comen a diario. Deben tener en cuenta los casos médicos con los que se esté trabajando en cada momento y que dieta es la que más beneficia al paciente en cada actividad. 


\section{Elementos de desarrollo curricular}

\section{Objetivos}

Los objetivos específicos que se pretende que el alumnado alcance a través de la realización del proyecto integrado son:

1) Comprender la organización y funcionamiento de un hospital.

2) Aplicar conocimientos académicos en un contexto que simula el ámbito laboral.

3) Trabajar aplicando el método científico.

4) Recordar de manera general los contenidos abordados durante cursos previos de la Educación Secundaria en las materias de Ciencias de la Naturaleza, Biología y Geología, y Física y Química, Matemáticas.

5) Trabajar de manera más profunda las características de los seres vivos y su composición.

6) Profundizar en conceptos de economía.

7) Usar el laboratorio en general y el microscopio, en particular.

8) Seguir los protocolos básicos sobre seguridad en el laboratorio.

9) Aplicar las nuevas tecnologías en el proceso de resolución de los problemas planteados.

10) Tomar decisiones fundamentadas consensuadas con el grupo de trabajo.

11) Argumentar, debatir y evaluar propuestas.

\section{Contenidos}

A través del proyecto integrado se abordarán los siguientes contenidos conceptuales (i-iv), procedimentales (v-vii) y actitudinales (viii-xii):

i. Características generales y composición del cuerpo humano.

ii. Conceptos básicos de la economía.

iii. Salud y medio ambiente.

iv. Generación y gestión de residuos sanitarios.

v. Interpretación de diferentes casos reales y ficticios aplicando las etapas del método científico.

vi. Análisis de información en diferentes formatos audiovisuales.

vii. Elaboración, interpretación y uso de gráficas, tablas, etc.,

viii. Valoración de la importancia del método científico para el avance de las ciencias de la salud.

ix. Valoración de la importancia de las ciencias en la vida cotidiana.

x. Valoración del orden y seguridad en el laboratorio.

xi. Valoración del impacto de la acción humana en el medio ambiente y como esto repercute en la salud de la sociedad.

xii. Resolución de conflictos y respeto a los componentes del grupo.

\section{Competencias}

En el proyecto integrado se favorece el desarrollo de todas las competencias clave indicadas en el Real Decreto 1105/2014, de 26 de diciembre, por el que se establece el currículo básico de la Educación Secundaria Obligatoria y del Bachillerato (Ministerio de Educación, Cultura y Deporte, 2015a), en las que profundice la Orden ECD/65/2015 (Ministerio de Educación, Cultura y Deporte, 2015b).

a) Competencia en comunicación lingüística. La comunicación es una actividad imprescindible en este proyecto, tanto entre los componentes del grupo para la 
toma de decisiones, como en la exposición del trabajo final, donde es especialmente importante la presentación, la expresión oral y el uso de vocabulario adecuado.

b) Competencia matemática y competencias básicas en ciencia y tecnología. El alumnado debe manejar datos, sobre todo en el grupo de trabajo de gestión administrativa. También es necesario hacer cálculos, elaborar e interpretar gráficas para llevar una adecuada gestión económica ajustada al presupuesto en cada caso. Por otro lado, el alumnado tendrá que elaborar razonamientos inductivos y deductivos, así como utilizar las metodologías científicas, ya que en la primera fase de la actividad se le facilitan unos datos y a partir de estos debe construir hipótesis, recabar información (lo que conlleva una investigación), sacar conclusiones, proponer posibles teorías y analizarlas.

c) Competencia digital. El proyecto promueve el uso de las nuevas tecnologías en la búsqueda de información, herramientas y recursos, haciendo que el alumnado tenga que desenvolverse y acceder a la oferta de servicios de la actual sociedad del conocimiento.

d) Competencia para aprender a aprender. Al plantearse nuevos retos, el alumnado será consciente de sus carencias y tendrá que superarlas mediante los aprendizajes convenientes, haciendo que las dificultades vayan disminuyendo conforme evolucione en el proyecto.

e) Competencias sociales y cívicas. Como se ha comentado, el proyecto está planteado para trabajar en tres grupos entre los cuales el alumnado se irá rotando y que deben estar en constante comunicación entre sí. Esto propicia el desarrollo de responsabilidades tanto individuales como grupales, pues cada participante estudiante debe aportar y cooperar en su grupo de trabajo. Para ello, los estudiantes deberán enfrentarse a la resolución de posibles conflictos con el resto de los compañeros y con los demás grupos.

f) Sentido de iniciativa y espíritu emprendedor. El trabajo en grupo es una buena estrategia para desarrollar esta competencia pues potencia las habilidades sociales al tener que estar el alumnado en constante comunicación. Esto también es positivo a la hora de adquirir actitudes como son la responsabilidad, la autocrítica y el liderazgo, entre otros.

g) Conciencia y expresiones culturales. A la hora de exponer el resultado de cada caso propuesto, el alumnado deberá apoyar su discurso con diferentes medios y materiales que le ayuden a defender su postura, por lo que se desarrollará esta competencia al poner en juego la creatividad de los participantes.

\section{Evaluación}

Se establecen como criterios para la evaluación del aprendizaje del alumnado los dados por los objetivos específicos del proyecto. Para una mayor concreción, los indicadores siguientes desarrollan los criterios de evaluación asociados a los objetivos específicos mostrados entre paréntesis:

- Relaciona las problemáticas propuestas dialogando sobre ellas con el resto de los miembros de la clase $(11,12)$.

- Escucha y respeta las opiniones de los compañeros y del profesorado $(11,12)$.

- Incorpora elementos novedosos que sean pertinentes para el tema propuesto (1).

- Recupera y poner en uso conocimientos diversos de manera interdisciplinar (3, $4,5,8)$.

- Utiliza adecuadamente las Tics en la resolución de las actividades $(8,9)$.

- Colaborar con el grupo de modo activo y cooperativo $(11,12)$.

- Comprende el Método Científico y lo aplica adecuadamente en las diferentes 
actividades $(2,3)$.

- Busca, recopila y procesa información $(4,9)$.

- Maneja fuentes de información (4, 5, 6,9).

- Usa vocabulario adecuado $(11,12)$.

- Integra el conocimiento matemático con otros tipos de conocimiento (2).

- Comprende los problemas (2).

- Usa los recursos tecnológicos disponibles $(7,9)$.

- Toma decisiones con respecto al uso sostenible $(1,2)$.

- Valora las ideas de los demás (12).

- Acepta los errores y aprende de los demás (12).

- Formula sus propios argumentos de manera adecuada $(11,12)$.

- Adquiere responsabilidades y compromisos personales (9).

- Compone producciones responsables y creativas (2).

- Reflexiona de forma crítica ante los problemas $(11,12)$.

- Identifica la diversidad de respuestas (12).

- Transforma la información en conocimiento propio (2).

- Aplica los conocimientos adquiridos en situaciones parecidas (2).

- Organiza tiempo y tareas (10).

\section{Actividades}

En este proyecto integrado se plantean tres tipos de actividades:

- La actividad de apertura, que será con la que se inicie el proyecto, es la toma de contacto y el inicio de todo el proceso que se pretende llevar a cabo durante el curso.

- Las actividades mensuales, que se adaptan a la temporalización de 4 sesiones por mes, son actividades-tipo en las que todos los grupos van a trabajar en diferentes temáticas pero llevando una misma línea. En el presente trabajo vamos a incluir 4 ejemplos de estas actividades.

- La actividad de cierre tiene como función principal que los estudiantes expongan al resto del centro el trabajo que han realizado desarrollando el proyecto.

Actividad 10: Apertura de un hospital. Esta actividad representa la actividad inicial que se propone al alumnado una vez explicado la dinámica de trabajo que va a suponer el proyecto integrado. Se plantea la apertura de un nuevo hospital, por lo que el alumnado es el responsable de la decisión de instalaciones y puesta a punto para que el hospital en el que trabajamos pueda abrir sus puertas. Se debe hacer una investigación para saber de que se compone un hospital y las necesidades mínimas que se tienen que cubrir. En esta actividad sería conveniente llevar a cabo una visita al hospital más cercano para que los estudiantes se empiecen a familiarizar con la realidad hospitalaria y les sea más fácil ubicar las necesidades a tener en cuenta. Por parte de cada grupo, los cuales ya se habrán establecido, se elaborará un listado de lo que se ha decidido, dependiendo de cada grupo, que hay que tener en cuenta para la apertura del hospital dependiendo del grupo al que se pertenezca. Entre las tareas a realizar por cada grupo se encuentran entre otras:

1. Grupo sanitario y de salud: chequeo al personal contratado: Pruebas a realizar en un chequeo laboral rutinario. Recursos: Ejemplo de chequeo médico laboral 
2. Grupo gestión ambiental. estudio de los residuos sólidos urbanos (RSU) generados antes de empezar la actividad sanitaria.

- Residuos generados

- Gestión de residuos

Recursos: Procedimiento de gestión de residuos en un hospital.

http://www.juntadeandalucia.es/servicioandaluzdesalud/hmotril/paginas/gestion_adm/s ervicios_generales/Control_residuos.pdf

3. Grupo gestión administrativa. Elaboración de contratos y nóminas para la plantilla.

Recursos: Guía para la elaboración de nominas y contratos laborales.

http://www.educa2.madrid.org/web/educamadrid/principal/files/3bdebad9-66e9-4155$87 \mathrm{bd}-$ 747807564054/Pasos\%20en\%20la\%20elaboraci\%C3\%B3n\%20de\%20la\%20nomina\% 20y\%20ejemplo\%20practico.pdf

http://transparencia.edomex.gob.mx/fidepar/informacion/manualprocedimientos/Proced imiento_Elaboracion_Contratos.pdf

4. Grupo de infraestructuras y mantenimiento

Establecimiento de las salidas de emergencias con las que debe contar un hospital.

Recursos: Normas básicas de seguridad en hospitales.

http://www.buenastareas.com/ensayos/Normas-Basicas-De-Seguridad-EnHospitales/1530025.html

5. Grupo de documentación médica. Generar una base de datos con el personal contratado.

Recursos: Como elaborar una base de datos de personal profesional.

http://www.excelnegocios.com/base-de-datos-personal-con-fotos/

6. Grupo de nutrición: Estudio de los hábitos alimenticios del personal.

Recursos: Ejemplo de un estudio alimenticio.

http://www.munideporte.com/imagenes/documentacion/ficheros/00da422f.pdf

Esta actividad servirá como base para las siguientes, ya que el alumnado estará familiarizado con instalaciones y servicios a los que se tienen que enfrentar en las situaciones propuestas en cada actividad. Junto con la actividad de cierre son las únicas que se salen del esquema establecido de cuatro sesiones en clase.

Actividad 20: Diagnóstico y tratamiento de una enfermedad infecciosa del sistema respiratorio, cambio de una caldera y del sistema de revelado de placas radiográficas. En las actividades que se realizan a lo largo del curso se les plantea al 
alumnado un enunciado que cuente con varios problemas, que afecten de a todos los grupos establecidos para que en cada actividad todo el alumnado pueda ser participes y siempre teniendo en cuenta que se debe trabajar desde la perspectiva sostenible y ambiental. Se va a presentar un caso con síntomas de tuberculosis pulmonar como principal problema. También se sugiere el cambio de una caldera y del sistema de revelado de placas radiográficas del hospital. A partir de esto, todos los grupos deberán trabajar tanto de manera independiente como en colaboración con los otros grupos para llevar a cabo el desarrollo de la actividad. Para ello, se les facilitan varios recursos diferentes dependiendo del grupo de trabajo. Estos recursos constituyen una ayuda para comenzar a trabajar en la actividad pero se sugerirá a los grupos la búsqueda de más material que les facilite la resolución del problema. Entre los problemas plantados está:

* Se presenta el caso de un hombre de 55 años, con pasado de alcoholismo y con estos síntomas:

- Una tos intensa que dura 3 semanas o más

- Dolor en el pecho

- Tos con sangre o esputo (flema desde el fondo de los pulmones)

- Debilidad o cansancio

- Pérdida de peso

- Falta de apetito

- Escalofríos.

- Fiebre

- Sudor durante la noche

* A su vez, el hospital cuenta con una caldera de gasoil la cual ha sufrido una avería imposible de arreglar, por lo que se debe sustituir por una caldera nueva y, aprovechando esta situación, se sugiere hacer el cambio siguiendo criterios sostenibles.

* El hospital en el que trabajamos todavía cuenta con un sistema de almacenamiento de placas reveladas, por lo que el espacio dedicado al archivo se está agotando y habría que buscar alternativas para solucionar el problema.

7. Grupo sanitario y de salud: Se debe decidir qué pruebas se van a realizar al paciente según los síntomas que presenta y a través de esto establecer el diagnostico y el tratamiento adecuado:

- Pruebas a realizar

- Diagnostico

- Tratamiento

Recursos: Webs que ayudan al diagnostico de enfermedades según los síntomas.

http://sintomas.com.es/

http://diagnosticodesintomas.com/

8. Grupo gestión ambiental. Establecer qué residuos se generan con las actividades a partir del ingreso del paciente y su correcta gestión.

- Residuos generados

- Gestión de residuos 
Recursos: Procedimiento de gestión de residuos en un hospital.

http://www.juntadeandalucia.es/servicioandaluzdesalud/hmotril/paginas/gestion_adm/s ervicios generales/Control_residuos.pdf

9. Grupo gestión administrativa. Elaboración de un presupuesto para el caso asignado teniendo en cuenta todas las actividades que se realizan tanto sanitarias como no sanitarias.

Recursos: Guía para la estimación de costes hospitalarios.

http://www.salud.gob.mx/unidades/cdi/documentos/DOCSAL7417.pdf

10. Grupo de infraestructuras y mantenimiento. Estudio de diferentes tipos de calderas y elección de las más eficiente.

Recursos: Guía básica de calderas industriales.

http://www.ingenieros.es//files//Proyectos_1/Guia-basica-calderas-industrialeseficientes-fenercom-2013.pdf

11. Grupo de documentación médica. Investigación sobre los tipos de tecnologías para el visionado de imágenes médicas. Propuesta de un nuevo sistema eficiente en almacenamiento y con mínimo impacto ambiental.

Recursos: Web con ejemplo de digitalización de historias médicas.

http://essalud.gob.pe/downloads/empresarial/salud/boltecno26.pdf

12. Grupo de nutrición. Diseño de dieta alimenticia adecuada al tipo de enfermedad diagnosticada. Elección de los alimentos en base al $\mathrm{CO}_{2}$ generado según el lugar de origen.

Recursos: Webs de nutrición, recetas y composición alimenticia.

http://www.bedca.net/bdpub/index.php

http://www.dimequecomes.com/

Actividad $3^{\circ}$ : Diagnóstico y tratamiento de una enfermedad no infecciosa, rotura de un tanque de gasoil, gestión del agua y almacenamiento de historias clínicas. Se va a presentar un caso con síntomas de leucemia como principal problema. En esta actividad se produce una rotura de un tanque de gasoil que alimenta al grupo electrógeno, por lo que hay que tener en cuenta los efectos ambientales y como se actúa ante esto. El agua en un hospital es muy importante y hay que tener un estricto control de su calidad para la seguridad de los pacientes y profesionales que allí trabajan, por lo que se debe tener un procedimiento de actuaciones preventivas en el tratamiento del agua. Las historias clínicas de los pacientes que pasan por el hospital suelen ocupar mucho espacio físico cuando se almacenan en papel y conlleva demasiado tiempo el traslado de los mismos cuando es solicitado por algún médico. Se recomienda una alternativa que sea más efectiva en los dos sentidos. Para ello, se les facilitan varios recursos diferentes dependiendo del grupo de trabajo. Estos recursos constituyen una ayuda para comenzar a trabajar en la actividad pero se 
sugerirá a los grupos la búsqueda de más material que les facilite la resolución del problema. Se presenta el caso de una mujer de 33 años, con estos síntomas:

- Sensación de cansancio

- Debilidad

- Mareo o aturdimiento

- Dificultad para respirar

- Fiebre.

- Infecciones recurrentes

- Sufrir moretones fácilmente

- Sangrado, como sangrado frecuente o grave de las encías o de la nariz

Se detecta que uno de los tanques de gasoil que alimenta las calderas de calefacción tiene una rotura por lo que se está produciendo un vertido en el suelo. El tanque es superficial y el vertido se observa a simple vista.

Las historias clínicas siguen archivándose en papel, lo cual hace que el espacio físico disminuya y que no sean accesibles en todo momento por los profesionales, se debe buscar alternativas.

1. Grupo médico. Se debe decidir qué pruebas se van a realizar al paciente según los síntomas que presenta y a través de esto establecer el diagnostico y el tratamiento adecuado:

- Pruebas a realizar

- Diagnostico

- Tratamiento

Recursos: Webs que ayudan al diagnostico de enfermedades según los síntomas.

http://sintomas.com.es/

http://diagnosticodesintomas.com/

http://www.cancer.org/

2. Grupo gestión ambiental. Establecer qué residuos se generan con las actividades a partir del ingreso del paciente y su correcta gestión. Establecer una respuesta ante emergencias ambientales, en este caso derrame de productos peligrosos.

- Residuos generados

- Gestión de residuos

- Derrame de combustibles

Recursos: Procedimientos de gestión de residuos y emergencias ambientales en un hospital.

http://www.juntadeandalucia.es/servicioandaluzdesalud/hmotril/paginas/gestion_adm/s ervicios_generales/Control_residuos.pdf

http://eies.ats.aq/Ats.IE/ContingencyFileUpload/Plan\%20contingencia\%20derrames\%2 0combustible-JCl.pdf 
3. Grupo gestión administrativa. Elaboración de un presupuesto para el caso asignado teniendo en cuenta todas las actividades que se realizan tanto sanitarias como no sanitarias.

Recursos: Guía para la estimación de costes hospitalarios.

http://www.salud.gob.mx/unidades/cdi/documentos/DOCSAL7417.pdf

4. Grupo de infraestructuras y mantenimiento. Elaborar un manual de procedimientos para el mantenimiento preventivo en el tratamiento de agua en un hospital.

Recursos: Guía para el mantenimiento de de aguas en un hospital.

http://bdigital.uao.edu.co/bitstream/10614/1139/8/TBM0267G.pdf

5. Grupo de documentación médica. Búsqueda de alternativas al archivo en papel para las historias clínicas.

Recursos: Webs con alternativas al archivo en papel.

https://www.msssi.gob.es/organizacion/sns/planCalidadSNS/docs/HCDSNS_Castellan o.pdf

http://www.biocom.com/sistema/historias_clinicas/historia_clinica_informatica.html

http://www.intramed.net/contenidover.asp?contenidolD=53769

6. Grupo de nutrición. Diseño de dieta alimenticia adecuada al tipo de enfermedad diagnosticada. Elección de los alimentos en base al $\mathrm{CO}_{2}$ generado según el lugar de origen.

Recursos: Webs de nutrición, recetas y composición alimenticia.

http://www.bedca.net/bdpub/index.php

http://www.dimequecomes.com/

Actividad 40: Diagnóstico y tratamiento de una enfermedad infecciosa del sistema nervioso, rotura de maquinaria de lavandería, diseño de protocolo de accidentes con material biológico y planificación de dietas personalizadas. En esta actividad encontramos síntomas en 3 pacientes adultos de meningitis como problema para el grupo sanitario. Además de establecer qué tipo de enfermedad presentan los pacientes se debe elaborar una dieta personalizada teniendo en cuenta el sexo, peso e historial clínico. Las agujas de kirschner son demasiado largas para los contenedores de punzantes estándar, por lo que se planteará el diseño y elección de un nuevo tipo de contendor para evitar el riesgo de accidente. Ha habido una avería en una de las lavadores de la lavandería con la que cuenta el hospital y habrá que decidir el tipo de maquinaria por el cual se sustituye. Se va proceder a la destrucción de material con información confidencial que ya no es requerido por el hospital, para ellos se deberá seguir ciertas pautas que no conlleven un incumplimiento en la Ley Orgánica de Protección de Datos de Carácter Personal de España. Para ello, se les facilitan varios recursos diferentes dependiendo del grupo de trabajo. Estos recursos constituyen una ayuda para comenzar a trabajar en la actividad pero se sugerirá a los 
grupos la búsqueda de más material que les facilite la resolución del problema. Se presenta el caso de tres adultos de 57, 35 y 30 años, con estos síntomas:

- Fiebre y escalofríos

- Nauseas y vómitos.

- Sensibilidad a la luz.

- Cuello rígido.

- Dolor de cabeza intenso.

El hospital cuenta con lavandería industrial propia y una de las lavadoras ha sufrido una avería importante por la que pierde agua en el proceso de lavado, se recomienda la rápida sustitución de la máquina para evitar que se dispare el consumo de agua en el centro. Para el ahorro de espacio y limpieza de la zona de archivos se recomienda la eliminación de documentación pero ésta contiene datos e información confidencial por lo que se deben seguir unas pautas para la correcta eliminación.

1. Grupo médico. Se debe decidir qué pruebas se van a realizar a los pacientes según los síntomas que presenta y a través de esto establecer el diagnostico y el tratamiento adecuado y que procedimiento se sigue si resulta ser infecciona:

- Pruebas a realizar

- Diagnostico

- Tratamiento

- Procedimiento ante enfermedades infecciosas.

Recursos: Webs que ayudan al diagnostico de enfermedades según los síntomas y los procedimientos a seguir según el tipo de enfermedad.

http://sintomas.com.es/

http://www.nlm.nih.gov/medlineplus/spanish/ency/article/000680.htm

http://www.fesemi.org/documentos/1354119963/publicaciones/protocolos/protocolosenfermedades-infecciosas.pdf

2. Grupo gestión ambiental. Incorporación al procedimiento de gestión de residuos de contenedores que respondan a los nuevos residuos generados. Establecer una respuesta ante accidentes laborales con sustancias orgánicas y punzantes. Además deberá hacerse cargo de todos los residuos generados

- Residuos generados

- Gestión de residuos

- Respuesta ante accidentes biológicos

Recursos: Procedimientos de gestión de residuos y accidentes laborales con material orgánico en un hospital.

http://www.juntadeandalucia.es/servicioandaluzdesalud/hmotril/paginas/gestion_adm/s ervicios_generales/Control_residuos.pdf

http://www.ispch.cl/sites/default/files/u5/Guia_Preventiva_Cortopunzantes.pdf

3. Grupo gestión administrativa. Elaboración de un presupuesto para el caso 
asignado teniendo en cuenta todas las actividades que se realizan tanto sanitarias como no sanitarias.

Recursos: Guía para la estimación de costes hospitalarios.

http://www.salud.gob.mx/unidades/cdi/documentos/DOCSAL7417.pdf

4. Grupo de infraestructuras y mantenimiento. Búsqueda y elección de nueva maquinaria para lavandería teniendo en cuenta criterios sostenibles. Hacer comparativas con diferentes modelos.

Recursos: Guía para el mantenimiento de lavadoras industriales y criterios de elección.

http://www.efameinsa.com/manuales.php

http://www.promateriales.com/pdf/HP-07-09.pdf

5. Grupo de documentación médica. Elaboración de procedimientos de destrucción de material confidencial.

Recursos: Procedimiento de manejo y destrucción de material confidencial.

http://gpd.sip.ucm.es/sonia/docencia/master/Trabajos\%20Alumnos/Destruccion\%20se gura\%20de\%20datos/DestruccionSeguraDatos.pdf

http://www.juntadeandalucia.es/servicioandaluzdesalud/hmotril/paginas/gestion_adm/s ervicios generales/IO\%2001\%20Instruccion $\% 200$ perativa $\% 20$ Gestion $\% 20$ de $\% 201$ a $\%$ 20Documentacion\%20Confidencial\%201.pdf

6. Grupo de nutrición. Diseño de dieta alimenticia adecuada al tipo de enfermedad diagnosticada en base a las características de los pacientes:

- Paciente 1: adulto de 57.años, sexo masculino, $150 \mathrm{~kg}$, historial de hipertensión.

- Paciente 2: adulto de 35 años, sexo femenino, $68 \mathrm{~kg}$, embarazada.

- Paciente 3: adulto de 30 años, sexo masculino, $85 \mathrm{~kg}$, no presenta otro tipo de complicaciones.

Recursos: Webs de nutrición, recetas y composición alimenticia.

http://www.bedca.net/bdpub/index.php

http://www.dimequecomes.com/

Actividad $5^{\circ}$ : Actuación ante accidente múltiple, procedimiento de prevención contra incendios, selección y compra de equipamiento de radiodiagnóstico y jornadas de nutrición sanitaria. En esta ocasión el hospital en el que trabajamos recibe el aviso de un accidente múltiple de coche con un herido grave, dos leves y un fallecido para lo cual se debe establecer el procedimiento de que se activa para atender a los afectados. En la planta cuarta del hospital ha habido un incendio que ha afectado a una habitación, no ha habido heridos ya que en ese momento no estaba ocupada y el fuego ha sido controlado con rapidez pero el mobiliario se ha visto 
afectado, sobre todo dos camas y mesillas con las que contaba la habitación, por lo que habrá que hacer una selección de nuevo mobiliario. Derivado de este accidente, y para prevenir en casos futuros, se propone elaborar un procedimiento de prevención contra incendios. La unidad de Rayos $X$ se va a ampliar incorporando el equipamiento necesario para la realización de tomografía axial computarizada (TAC), por lo que se tienen que seguir ciertos criterios económicos y sostenibles para la elección. También se debe tener en cuenta la contratación de nuevo personal para el manejo del nuevo equipamiento. El hospital está organizando unas jornadas de nutrición sanitaria con productos de la tierra, estas jornadas van a durar una semana y deben contar con productos que pertenezcan a la zona y que sean de temporada. Una vez que contamos con espacio en la zona de archivo de historias clínicas se pretende reorganizar las historias clínicas físicas que hay archivadas en el hospital para lo cual se debe elaborar un procedimiento con las pautas de archivo y clasificación que vamos a seguir. Para todo esto se va a facilitar recursos diferentes dependiendo del grupo de trabajo. Estos recursos constituyen una ayuda para comenzar a trabajar en la actividad pero se sugerirá a los grupos la búsqueda de más material que les facilite la resolución del problema. Se presenta el caso de tres adultos y un niño con heridas de accidente de tráfico:

- Herido 1: hombre de 48 años. Fallecido en el acto.

- Herido 2: mujer de 46 años. Presenta síntomas de piernas rotas y fuerte golpe en la cabeza, no se tiene certeza de heridas internas. Está consciente en el momento del ingreso.

- Herido 3: hombre de 32 años. Presenta síntomas de brazo derecho roto y fuerte golpe en el tórax, no se tiene certeza de heridas internas. No está consciente en el momento de ingreso.

- Herido 4: niño de 11 años. Presenta síntomas de herida en la frente, no se tiene certeza de heridas internas. Está consciente en el momento del ingreso.

1. Grupo médico. Se debe decidir qué pruebas se van a realizar a los pacientes según los síntomas que presenta y a través de esto establecer el diagnostico y el tratamiento. También se va a elaborar un protocolo de cómo actuar ante un accidente de tráfico para los usuarios:

- Pruebas a realizar

- Diagnostico

- Tratamiento

- Procedimiento ante urgencias.

Recursos: Guía de actuación ante accidentes de tráfico.

http://nervion.us.es/DGT/GUIA_DIDACTICA_ACTUACION_ANTE_EL_ACCIDENTE_D E_TRAFICO.PDF

2. Grupo gestión ambiental. Establecer una respuesta ante incendios. Además deberá hacerse cargo de todos los residuos generados

- Residuos generados

- Gestión de residuos

- Procedimientos de prevención de incendios.

Recursos: Procedimientos de prevención contra incendios en un hospital.

http://www.hospitalrancagua.cl/antigua/calidad/Calidad\%20y\%20Seguridad/8- 
Seguridad\%20de\%20las\%20Instalaciones/ambito8/INS\%201.1\%20-

\%20Plan\%20Prevencion\%20de\%20Incendios\%20HRR\%20V0-2012.pdf

3. Grupo gestión administrativa. Elaboración de un presupuesto para el caso asignado teniendo en cuenta todas las actividades que se realizan tanto sanitarias como no sanitarias. Contratación de nuevo personal para la unidad de radiodiagnóstico.

Recursos: Guía para la estimación de costes hospitalarios.

http://www.salud.gob.mx/unidades/cdi/documentos/DOCSAL7417.pdf

4. Grupo de infraestructuras y mantenimiento. Búsqueda y elección de nuevas camas para las habitaciones teniendo en cuenta criterios sostenibles. Hacer comparativas con diferentes modelos.

Recursos: Guía sobre tipos de camas para hospital.

http://www.mcgraw-hill.es/bcv/guide/capitulo/8448177002.pdf

5. Grupo de documentación médica. Elaboración de un procedimiento de archivo de codificación de historias clínicas.

Recursos: Procedimiento de archivo de historias clínicas

file:///C:/Users/mdm/Downloads/GEDSA.\%20ORDENACI\%C3\%93N\%20Y\%20CODIFI CACI\%C3\%93N\%20DE\%20LAS\%20HISTORIAS\%20CL\%C3\%8DNICAS..pdf

http://www.archivo.palmira.unal.edu.co/paginas/documentos/Guia_Historias_Clinicas.p df

6. Grupo de nutrición. Preparación de las jornadas sanitarias con productos propios de la zona y de temporada. Hay que elaborar un menú diario durante siete días que cumpla los requisitos establecidos.

Recursos: Webs de nutrición, recetas y composición alimenticia.

http://www.bedca.net/bdpub/index.php

http://www.dimequecomes.com/

Actividad de cierre: La actividad de cierre coincidirá con el periodo final del curso escolar, por lo que se propone un cambio en la rutina de actividades. En esta ocasión se va a preparar unas jornadas de puertas abiertas tanto para el resto de compañeros del centro como para padres y personas interesadas, junto con la exposición del trabajo realizado durante el curso por el alumnado. El trabajo será expuesto cronológica conforme se ha ido trabajando con las actividades, pues se pretende que quede constancia de cómo se interrelacionan todas las actividades que se producen en hospital, siendo de tan variada naturaleza, y como trabajan en conjunto para llegar a un mismo objetivo, que en este caso en particular es el tratamiento y cura del paciente y todo visto desde una perspectiva ambiental y sostenible. Como hemos indicado anteriormente, esta actividad se sale de la temporalizarían de 4 sesiones establecidas. Estas se utilizarán para la elaboración de murales explicativos de todo lo 
realizado durante el curso escolar, también se utilizaran el material elaborado durante todo el proyecto como presentaciones, podcast, videos, etc. Estas jornadas tendrán lugar a lo largo de dos semanas, en el horario de recreo escolar para facilitar el acceso al resto de estudiantes del centro. El alumnado que ha participado en la elaboración del proyecto integrado será el encargado de ejercer de guía en las jornadas.

\section{Reflexión final}

La metodología de enseñanza basada en proyectos posibilita que el alumnado dirija y condicione la evolución de su propio aprendizaje, fomentando que los adolescentes adquieran una madurez propia a su edad y se incorporen a la vida adulta en condiciones de aplicar sus conocimientos, argumentar sus opiniones e integrarse positivamente en la sociedad.

La implementación del proyecto que se propone en este trabajo beneficiará al alumnado debido a su carácter interdisciplinar, pues integra conocimientos científicos con otros técnicos de otras disciplinas (contabilidad y economía), conciencia social y ambiental, utilizando elementos que se han aprendido en distintas materias a lo largo de todo el periodo educativo, y favorece el desarrollo de futuros proyectos laborales 0 académicos donde se puedan retomar las competencias ya adquiridas.

\section{Referencias}

Díaz, B.F. y Hernández, R.G. (1999). Estrategias docentes para un aprendizaje significativo. Una interpretación constructivista. México: Mc Graw Hill.

Consejería de Educación (2008). ORDEN de 5 de agosto de 2008, por la que se desarrolla el currículo correspondiente al Bachillerato en Andalucía. Boletín Oficial de la Junta de Andalucía, 26 de agosto de 2008, 98-222. Sevilla.

Hernández de La Torre, E. (2010). Cambiar e innovar en educación secundaria: los "proyectos integrados" para construir un conocimiento compartido. Enseñanza \& Teaching, 28(2), 79-95.

La Cueva, A. (2006). La enseñanza por proyectos: ¿mito o reto? En: S. Campos (Ed.), Ciencias. Antología. Primer Taller de Actualización sobre los Programas de Estudio 2006. Reforma de la Educación Secundaria (pp. 15-23). México D.F.: Secretaría de Educación Pública ed.

Lozano, J. (1998). Proyectos Integrados de aprendizaje como respuesta educativa a la diversidad. Revista Enseñanza: anuario interuniversitario de didáctica, 16, 231-248.

Ministerio de Educación, Cultura y Deporte (2015a). Real Decreto 1105/2014, de 26 de diciembre, por el que se establece el currículo básico de la Educación Secundaria Obligatoria y del Bachillerato. Boletín Oficial del Estado, 3 de enero de 2015, 169-546. Madrid.

Ministerio de Educación, Cultura y Deporte (2015b). Orden ECD/65/2015, de 21 de enero, por la que se describen las relaciones entre las competencias, los contenidos y los criterios de evaluación de la educación primaria, la educación secundaria obligatoria y el bachillerato. Boletín Oficial del Estado, 29 de enero de 2015, 69867003. Madrid.

Ministerio de Educación, Cultura y Deporte (2013). Ley Orgánica 8/2013, de 9 de diciembre, para la mejora de la calidad educativa. Boletín Oficial del Estado, 10 de Diciembre de 2013, 97858-97921. Madrid.

Porlán R. y Martín R. (2008). ¿Qué formación docente, para qué enseñanza de las ciencias? Cuadernos de Pedagogía, 384, 58-61.

Pozo, J. I. (1992). El aprendizaje y la enseñanza de hechos y conceptos. En C. Coll, J. I. Pozo, B. Sarabia y E. Valls (Ed.), Los contenidos en la Reforma. Enseñanza y aprendizaje de conceptos, procedimientos y actitudes (pp. 297-313). Madrid: Santillana/Aula XXI. 